\title{
Identification of protease $m 1$ zinc metalloprotease conferring resistance to deltamethrin by characterization of an AFLP marker in Culex pipiens pallens
}

FF Zou, Q Guo, Y Sun, D Zhou, MX Hu, HX Hu, BQ Liu, MM Tian, XM Liu, XX Li, L Ma, B Shen * and CL Zhu*

\begin{abstract}
Background: Continuous and excessive application of deltamethrin (DM) has resulted in the rapid development of insecticide resistance in Culex pipiens pallens. The quantitative trait loci (QTL) responsible for resistance to DM had previously been detected in CX. pipiens pallens. But locating the QTLs on the chromosomes remained difficult. An available approach is to first characterize DNA molecular markers linked with the phenotype, and then identify candidate genes.
\end{abstract}

Methods: In this study, the amplified fragment length polymorphism (AFLP) marker L3A8.177 associated with the QTL, was characterized. We searched for potential candidate genes in the flank region of L3A8.177 in the genome sequence of the closely related $C x$. pipiens quinquefasciatus and conducted mRNA expression analysis of the candidate gene via quantitative real-time PCR. Then the relationship between DM resistance and the candidate gene was identified using RNAi and American CDC Bottle Bioassay in vivo. We also cloned the ORF sequences of the candidate gene from both susceptible and resistant mosquitoes.

Results: The genes CYP6CP1 and protease $m 1$ zinc metalloprotease were in the flank region of L3A8.177 and had significantly different expression levels between susceptible and resistant strains. Protease $m 1$ zinc metalloprotease was significantly up-regulated in the susceptible strains compared with the resistant and remained over-expressed in the susceptible field-collected strains. For deduced amino acid sequences of protease $\mathrm{m} 1$ zinc metalloprotease, there was no difference between susceptible and resistant mosquitoes. Knockdown of protease $m 1$ zinc metalloprotease not only decreased the sensitivity of mosquitoes to DM in the susceptible strain but also increased the expression of CYP6CP1, suggesting the role of protease $m 1$ zinc metalloprotease in resistance may be involved in the regulation of the P450 gene expression.

Conclusion: Our study represents an example of candidate genes derived from the AFLP marker associated with the QTL and provides the first evidence that protease $m 1$ zinc metalloprotease may play a role in the regulation of DM resistance in CX. pipiens pallens.

Keywords: Protease $m 1$ zinc metalloprotease, Deltamethrin resistance, Culex pipiens pallens, AFLP, CYP6CP1

\footnotetext{
* Correspondence: shenbo@njmu.edu.cn; clzhu@njmu.edu.cn

Department of Pathogen Biology, Nanjing Medical University, 140 Hanzhong

Road, Nanjing, Jiangsu 210029, PR of China
} 


\section{Background}

Vector-borne diseases account for over $17 \%$ of all infectious diseases, causing more than 1 million deaths annually [1]. Mosquitoes, the best known disease vectors, transmit diseases including malaria, dengue, yellow fever, West Nile fever and filariasis [2]. Currently, there are no commercially available vaccines against malaria, West Nile fever and filariasis so vector control is considered important for the control of diseases, and the core strategies of it mainly rely on insecticides [3]. There were four classes of insecticides recommended by the WHO, namely organochlorines, organophosphates, carbamates and pyrethroids [4], and only pyrethroids were allowed for use on long-lasting insecticidal nets (LLINs) [5]. Deltamethrin (DM), an important synthetic pyrethroid insecticide, has been accorded wider acceptance during the past few years due to its high activity against insects, environmental safety and high vulnerability to enzymatic degradation [6]. Unfortunately, many countries rely heavily on the use of insecticides in controlling mosquito vectors. As a result, vector control campaigns in some areas are facing serious problems with the rise of insecticide resistance, which has become a major obstacle for mosquito control. Effective insecticide resistance management (IRM) is essential, and determination of the mechanisms underpinning insecticide resistance will greatly assist the development of much-needed novel strategies for IRM.

Resistance to pyrethroids is a complex polygenic phenotype and the two best understood mechanisms of insecticide resistance in mosquitoes are target site insensitivity and metabolic resistance [7-9]. A number of genes associated with insecticide resistance were reported, including cytochrome P450s, esterases, GST, sodium channel gene, G-protein-coupled receptor-related genes and serine proteases [10-12]. Indeed, none of the currently known genes can entirely explain the molecular basis for pyrethroid resistance. Moreover, insecticide exposure is a potent selective force and insecticide resistance is evolving. Identifying novel genes associated with pyrethroid resistance is critical for the effective control of mosquitoes.

A preliminary study using $\mathrm{F}_{2}$ progeny from crossing between a susceptible and a resistant strain of $C x$. pipiens pallens identified seven deltamethrin-related QTLs. 12 AFLP markers flanking QTLs, in linkage with the resistance gene were cloned and blasted to a unique genomic position of the $C x$. pipiens quinquefasciatus reference genome (supercontigs) [13]. Cx. pipiens quinquefasciatus and $C x$. pipiens pallens are the two most common subspecies in the $C x$. pipiens complex. Introgression between these species is common in the United States and the taxonomic status of this complex has been a subject of debate [14, 15].
The Cx. pipiens quinquefasciatus genome sequence was recently determined using the whole genome shotgun approach, thus providing a valuable resource for advancing genome studies in this species complex [16]. As the genome assembly of $C x$. pipiens quinquefasciatus is represented by a high number of supercontigs with no order or orientation on the chromosomes. Locating the QTLs on the chromosomes remained difficult. An available approach is sequencing molecular markers associated with the QTL, then isolating and characterizing flanking genomic region [17]. In the present study, the genomic sequence surrounding the sequenced AFLP markers were used to search for candidate genes potentially related to insecticide resistance. Marker L3A8.177 in the vicinity of DR-4 (the QTL contributing $15 \%$ to phenotypic variation) matched a noncoding region in the supercontig 3.388. Two candidate genes were found around the genomic sequence surrounding AFLP markers, CYP6CP1 (VectorBase ID CPI J012484; 119,517 bp away from the L3A8.177) and protease $m 1$ zinc metalloprotease (VectorBase ID CPIJ01 2471; 386,339 bp from the L3A8.177), which were significantly differently expressed between susceptible and resistant strains of $C x$. pipiens pallens. The CYP6CP1 belongs to a member of CYP6 family, and there are many reports demonstrating the relationship between pyrethroid resistance and elevated activity of CYP6 family members in different mosquito species [18]. The protease $m 1$ zinc metalloprotease belongs to families of zinc metalloproteases, involved in a variety of physiologically important processes such as protein processing and turnover, regulation of peptide hormone action, viral infection, tissue invasion, and cell cycle control $[19,20]$. Moreover, the relationship between metalloproteases and biological insecticide resistance had been reported. Paris et al. identified a gene coding for an aminopeptidase potentially involved in resistance to $\mathrm{Ba}$ cillus thuringiensis israelensis (Bti) toxins in mosquitoes by combining AFLP genome scans and 454 pyrosequencing [21]. And Lee et al. reported that aminopeptidases were altered in an insecticide resistant strain of Aedes aegypti using transcriptome sequencing [22]. As the association of protease $m 1$ zinc metalloprotease with deltamethrin resistance in mosquito had not been reported, we focused on the study of protease $m 1$ zinc metalloprotease.

We characterized protease $m 1$ zinc metalloprotease in DM-resistant strains and susceptible strains in both laboratory and field populations. The expression profiles of protease $m 1$ zinc metalloprotease in different mosquito life stages was also established. RNA interference strategy was used in susceptible mosquitoes to investigate the relationship between protease $m 1$ zinc metalloprotease and DM resistance. The protease $m 1$ 
zinc metalloprotease knockdown not only decreased the sensitivity of mosquito to DM but also increased the expression of CYP6CP1, suggesting that protease $m 1$ zinc metalloprotease may regulate mosquito DM resistance through modulating detoxification metabolism.

\section{Methods}

\section{Mosquito strains}

Several laboratory-reared strains of $C x$. pipiens pallens were used in this study: a deltamethrin-susceptible (LabDS) strain (the $50 \%$ lethal concentration $\mathrm{LC}_{50}=0.04 \mathrm{mg} /$ L) and 4 deltamethrin-resistant (Lab-DR) strains of different resistance levels (Lab-DR1, Lab-DR2, Lab-DR3, LabDR4). The $\mathrm{LC}_{50}$ of $4 \mathrm{Lab}-\mathrm{DR}$ strains was $0.31 \mathrm{mg} / \mathrm{L}$, $0.85 \mathrm{mg} / \mathrm{L}, 3.03 \mathrm{mg} / \mathrm{L}, 3.43 \mathrm{mg} / \mathrm{L}$, respectively. The LabDS strain was collected from Tangkou town of Shandong Province (35.12 N; $116.50 \mathrm{E})$ in 2009 and reared without exposure to any insecticide. The Lab-DR strains were selected from the Lab-DS strain, and the selection detail was showed previously $[13,23]$. In addition, three samples were made of various structures in female adults (leg, head, thorax, abdomen). The individuals were obtained over all life stages from both Lab-DS and Lab-DR4 strains. We also collected five field populations of $C x$. pipiens pallens from Shangdong Province: PY (Pingying City, 36.29 N; 116.42E), SH (Shanghe City, 37.31 N; 117.15E), GD (Gudao City, 37.85 N; 118.81E), HM (Huiming City, 37.45 N; 117.41E), JN (Jining City, 35.26 N; 116.35E). For each field mosquito population, larvae were brought back to the insectary after morphological identification. To distinguish susceptible and resistant strains of field populations, female adults were exposed to discriminating doses of deltamethrin $(0.05 \%)$ for susceptibility tests following the standard WHO testing protocol [24]. The mosquitoes that survived the 24-h recovery period were classified as DM resistant, while those knocked down in an hour during the bioassay were classified as DM susceptible. All mosquito samples were preserved for further analysis at $-80{ }^{\circ} \mathrm{C}$.

\section{RNA extraction, CDNA synthesis and quantitative real- time PCR analyses}

Total RNA was extracted from all mosquitoes (Lab-DS, Lad-DR1, Lab-DR2, Lab-DR3 and Lab-DR4; susceptible and resistant ones of five field strains). The method of RNA extraction and cDNA synthesis was as the description of Zhou et al. [25].

All quantitative real-time PCR (qRT-PCR) analysis mentioned in the article was as the following description. The experimental technique was performed on the ABI PRISM 7300 (Applied Biosystems, USA) using Power SYBR Green PCR Master Mix (Applied Biosystems, USA) according to the manufacturer's protocol. The qRT-PCR primers were designed using Primer 5.0
(Additional file 1), based on the corresponding cDNA sequences obtained from the VectorBase. The qRTPCR analysis was performed using the method described previously [26]. The PCR products were used for melting curve and agarose gel electrophoresis analysis to confirm their amplification specificity. The assays were conducted in triplicate, and the average value of the triplicate was used for analysis of gene expression differences. The $\beta$-actin and RsP7 genes were used as the standard for expression normalization for each gene because of their stable expression [27, 28].

\section{RNAi analysis and American CDC bottle bioassay}

Double-stranded RNA (dsRNA) for two genes and negative control (NC) RNA were designed and synthesized from GenePharma (GenePharma, Shanghai, China) at a concentration of $20 \mu \mathrm{M}$ (The sequences were in Additional file 2, siRNA404 for CYP6CP1, siRNA345 for protease $m 1$ zinc metalloprotease). Approximately $350 \mathrm{ng}$ of dsRNA or NC RNA was injected into 1-day-old female mosquitoes (siR NA404 into the Lab-DR4 strain, siRNA345 into the LabDS strain). The procedure of microinjection on RNAi assays were carried out according to standard methodology $[29,30]$. For one biological replicate, 30 female adults were injected with dsRNA, 30 were injected with NC RNA, and another 30 were non-injected. Three biological replicates were performed. The groups were kept in the insectary at $28-30{ }^{\circ} \mathrm{C}$ in a 16 -h light/8-h dark photoperiod with 70 $80 \%$ humidity for 3 days. Then we verified the RNAi efficiency using qRT-PCR and detected the relative mortality of adult female mosquitoes under DM exposure by American CDC bottle bioassay. We conducted the CDC bottle bioassay as a guideline for evaluating insecticide resistance in vectors. (http://www.cdc.gov/parasites/education_training/lab/bottlebioassay.html). The diagnostic doses of DM applied in this study were $4 \mathrm{mg}$ for Lab-DR4 and $0.01 \mathrm{mg}$ for Lab-DS, per bottle $(250 \mathrm{~mL})$ of $20-25$ individuals. The control bottle coated with acetone was with dsRNA-microinjected individuals. The number of dead mosquitoes was recorded every $15 \mathrm{~min}$, up to $2 \mathrm{~h}$. At last the mortality rate was calculated at the diagnostic time.

\section{Cloning and sequencing for protease $m 1$ zinc metalloprotease}

The open reading frame (ORF) of protease $m 1$ zinc metalloprotease was amplified using a pair of specific primers: forward primer: 5' ATGAACAACAGCAAC AACGCCAAGA-3' and reverse primer: 5' CTACGG GTACTTTTCTGGCATAGTT-3', which were designed based on the cDNA sequence of $C x$. pipiens quinquefasciatus (https://www.vectorbase.org/Culex_quinquefasciatus/ Transcript/Sequence_cDNA?db=core;g=CPIJ012471). PCR was performed using PrimeSTAR ${ }^{\circ}$ HS DNA Polymerase (Code No. R010A, TaKaRa, Japan) following the 
manufacturer's recommendations in both Lab-DS and Lab-DR4 strains. Amplified products were electrophoresed on a $1 \%$ agarose gel, and target bands were excised and purified using TaKaRa MiniBEST Agarose Gel DNA Extraction Kit Ver. 4.0 (Code No. 9762, TaKaRa). Purified product was cloned into the pMD-19 T Simple Vector. Recombinant plasmid DNA was isolated and sequenced by the Beijing Genomic Institute. Sequences were accepted if identical from three different individuals from one strain.

\section{Statistical analysis}

The statistical significance of the gene expression was calculated using a Student's t-test [31] and mosquito mortality was analyzed using the chi-square test [32], by the Social Sciences (SPSS) software. Linear regression analysis was used to correlate transcription level and DM resistance level $\left(\mathrm{LC}_{50}\right)$ in $C x$. pipiens pallens [25, 31]. A value of $P<0.05$ was considered statistically significant.

\section{Results and discussion}

Screening of candidate genes in the flank of the marker The AFLP marker L3A8.177 matched a non-coding region in the supercontig 3.388 (3.388:393,117-393,273; Score $=308$, Except $=9 \mathrm{e}-74$, Identity $=99.37 \%$ ) [13] . There were 18 genes in the flank region of the marker in supercontig 3.388 (Additional file 3). By combination the molecular functions and biological processes of genes (https://www.vectorbase.org/search) with a keyword search (insecticide resistance) in the NCBI database (http:// www.ncbi.nlm.nih.gov/pubmed/), we selected five genes (CPIJ012485, CPIJ012484, CPIJ012475, CPIJ012472 and CP IJ012471) as potential candidate genes.

The transcription profiles of five genes associated with the marker were examined using qRT-PCR. As shown in Fig. 1, the Lab-DR4 strain $\left(\mathrm{LC}_{50}=3.43 \mathrm{mg} / \mathrm{L}\right)$ had a higher expression level for CPIJ012484 (CYP 6CP1) $\left(t_{(4)}=4.34, P=0.006\right)$, while a lower expression level for CPIJ012471 (protease $m 1$ zinc metalloprotease) $\left(t_{(4)}=51.23, P<0.0001\right)$, compared to the Lab-DS strain. Moreover, this result matched the report by Lv et al., that the range of expression level between the resistant and susceptible strain was a 1.3-fold difference for CYP6CP1, and 0.75 -fold for protease $m 1$ zinc metalloprotease using comparative transcriptome analyses by RNA sequence in Cx. pipiens pallens [10].

In recent years, the availability of tightly linked robust PCR-based markers has been used extensively to map and tag candidate genes of quantitative traits in many species. For example, Lyons et al. characterized AFLP markers associated with growth in Marsupenaeus japonicus, and identified a candidate gene surrounding the AFLP band 7.21a [17]. And Podio et al. obtained the flanking regions of a group of molecular markers linked

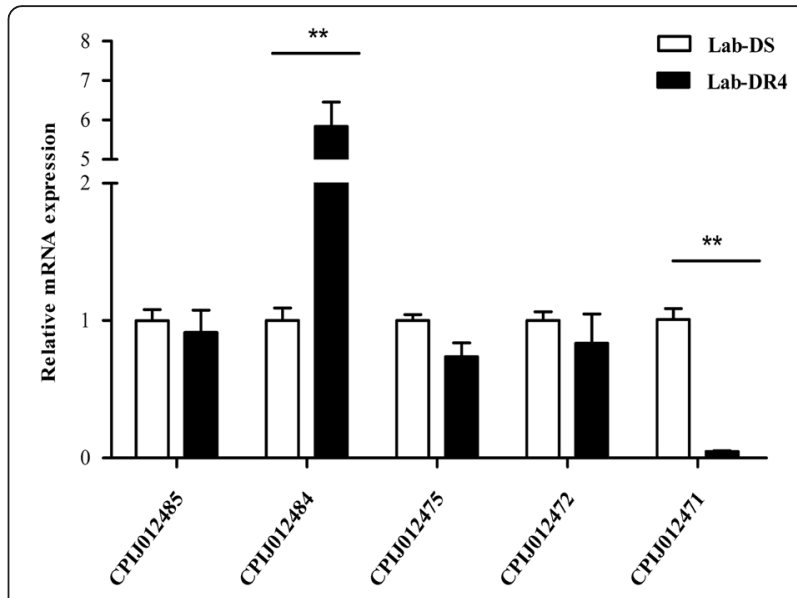

Fig. 1 Screening potential candidate genes via quantitative realtime PCR in Culex pipiens pallens. The relative expression levels of five genes were detected in adult female mosquitoes of the LabDS $\left(L C_{50}=0.04 \mathrm{mg} / \mathrm{L}\right)$ and $\mathrm{Lab}-\mathrm{DR} 4\left(L C_{50}=3.43 \mathrm{mg} / \mathrm{L}\right)$ strains. The gene CPIJ012484 (CYP6CP1) was significantly over-expressed in the resistant strain while gene CPIJ012471 (protease m1 zinc metalloprotease) was under-expressed. The results were shown as the mean \pm S.E. ${ }^{* *} P<0.01$ compared with the Lab-DS strain

to apospory, in order to identify possible candidate genes in Paspalum notatum [33]. In a previous report, we obtained genomic positions of several AFLP markers linked to QTLs referring DM resistance in the Cx. pipiens quinquefasciatus reference genome. And here we obtained two genes in the flank region of the marker L3A8.177, showing the different expression levels among Lab-DS and LabDR4 strains. Our study demonstrated AFLP marker is a relatively efficient marker for the genetic architecture analysis of quantitative traits.

Our finding detected that $C Y P 6 C P 1$ had a significantly higher expression in the resistant strain compared with the susceptible strain. To date, increased P450-mediated detoxification has been found in many insect species associated with enhanced metabolic detoxification of insecticides, by the increased levels of P450 proteins and P450 activity that result from constitutively transcriptional over expression of $\mathrm{P} 450$ genes in insecticide resistant insects [9, 34-37]. And the members of CYP6 family have been verified to be involved in pyrethroid metabolism [23, 38-40]. The result of CYP6CP1 was in keeping with the verification. However, protease $m 1$ zinc metalloprotease was only reported to be differently expressed between susceptible and resistant mosquitoes by the transcriptom studies, and we chose the gene for further study.

\section{In vivo validation by RNAi and American CDC bottle bioassay}

To evaluate the involvement of protease $m 1$ zinc metalloprotease in DM resistance in vivo, we sought to specifically 
inhibit the expression of the gene by injecting its corresponding dsRNA into the thorax of the adult female mosquitoes. The expression of protease $m 1$ zinc metalloprotease decreased $50 \%$ by siRNA345, compared with that in the corresponding NC group, separately $\left(t_{(4)}=3.19\right.$, $P=0.016$ ) (Additional file 4). The consequence of decrease in gene transcript on the susceptibility of female adults against DM was examined by the American CDC Bottle Bioassay (Fig. 2). For protease $m 1$ zinc metalloprotease in the Lab-DS strain, the siRNA345 group had a lower mortality rate compared to the other groups. And knockdown of the gene decreased the sensitivity of mosquitoes to DM.

\section{Cloning the ORF sequence of protease $m 1$ zinc metalloprotease}

We cloned the ORF regions of protease $m 1$ zinc metalloprotease in Lab-DS and Lab-DR4 strains. The ORF region has $924 \mathrm{bp}$ and encodes for a protein with 308 amino acids (Additional file 5). The protease $m 1$ zinc metalloprotease of $C x$. pipiens pallens shared the highest homology with Cx. pipiens quinquefasciatus (94\% identity) by the standard protein/protein BLAST sequence comparison program (http://beta.uniprot.org/?tab=blast). An ORF sequence analysis carried out in two strains showed that only point mutation was found at the 806th base, and the mutation $(806 \mathrm{~T}>\mathrm{C})$ was synonymous. The CDD software for structure analysis (http://www.ncbi.nlm.nih.gov/Structure/cdd/wrpsb.cgi) was used, demonstrating that the putative protein contains a domain: M1_APN_2 domain, from positions 150th to 291th (E-value: 2.16e-21).
The protease $m 1$ zinc metalloprotease is a member of the peptidase family $\mathrm{M}$. It preferentially cleaves neutral amino acids from the $\mathrm{N}$-terminus of oligo peptides and is present in a variety of tissues and cell types [41]. And the family has been identified in many parasites and vectors. For example, zinc-metalloprotease GP63 of Leishmania, a critical virulence factor, has been suggested to modulate cellular signaling through the subversion of host protein tyrosine phosphatase (PTP) function [42], significantly inhibits NLRP3 inflammasome function and IL-1 $\beta$ production [43]. In insects, aminopeptidase Ns (APNs) were also putative Cry toxin receptors (Cry proteins are pore-forming toxins that bind to the midgut epithelial cell membrane of susceptible insect larvae, causing extensive damage) [44-46]. In our study, the protease $m 1$ zinc metalloprotease gene of the Lab-DS strain exhibited a significantly higher mRNA level than the highly resistant strain. The injection of dsRNA corresponding to the gene sequence resulted in a significant gene knockdown effect in the susceptible strain, as evidenced by the presence of a significantly lower susceptibility to DM than in the control groups. But for deduced amino acid sequences of protease $m 1$ zinc metalloprotease, there was no difference between susceptible and resistant mosquitoes. The understanding of how resistance evolves at the molecular level is known predominantly to be involved in amplification, overexpression, and coding sequence variation of genes related to mechanisms of insecticide resistance [18]. These data strongly suggested that the role of protease $m 1$ zinc metalloprotease in $C x$. pipiens pallens resistance to DM may be by the change of the transcription level.

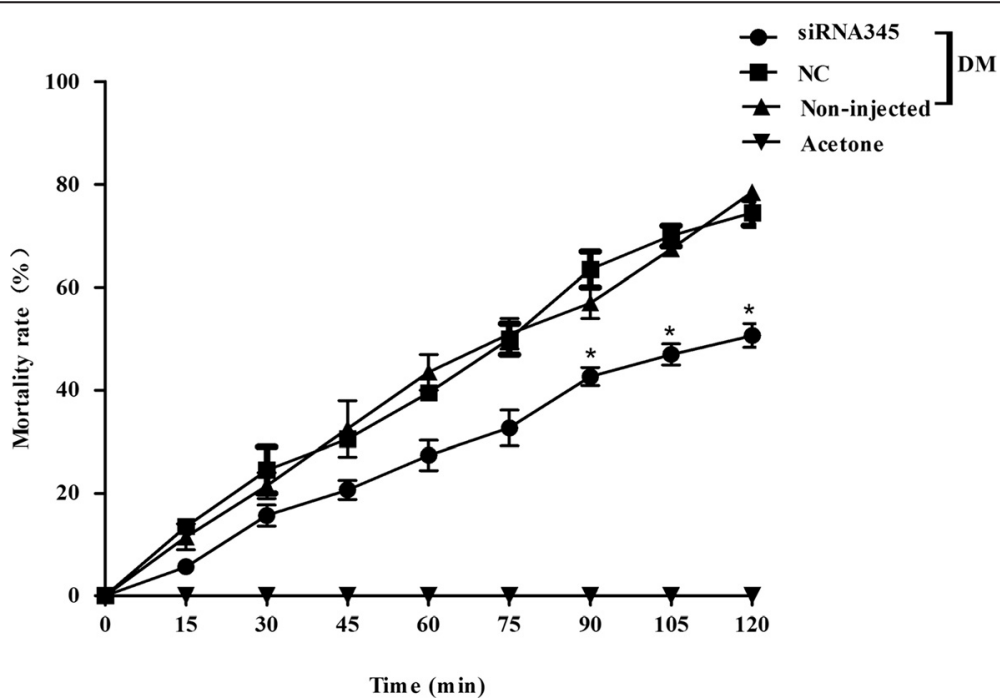

Fig. 2 Functional study of protease $m 1$ zinc metalloprotease in the Lab-DS strain. Mortalities of microinjected mosquitoes were observed after a $2 \mathrm{~h}$ exposure to American CDC bottles treated with deltamethrin $(0.01 \mathrm{mg} / \mathrm{ml})$. The siRNA345 microinjected group had a relatively lower mortality rate than the negative control $(\mathrm{NC})$ and non-injected groups. The figures show the mean \pm SD of three independent experiments $(* P<0.05)$ 


\section{Expression level analysis for protease $m 1$ zinc metalloprotease}

Over the life cycle of the mosquitoes, the relative RNA expression levels at all developmental stages (egg, L1-L4 instar larvae, pupae, male and female) of Cx. pipiens pallens were given in Fig. 3. The gene was transcribed at all stages but most in egg. And the expression levels were relatively low in larvae. It was worth mentioning that protease $m 1$ zinc metalloprotease was significantly upregulated at different stages (egg: $t_{(4)}=3.66, P=0.029$; L1: $t_{(4)}=6.96, P=0.002 ;$ L2: $t_{(4)}=3.29, P=0.030 ; \quad$ L3: $t_{(4)}=6.81, P=0.019 ;$ L4: $t_{(4)}=3.02, P=0.039$; pupae: $t_{(4)}=5.41, P=0.006$; female: $\left.t_{(4)}=51.23, \quad P<0.0001\right)$, except for the male adult, in the Lab-DS strain compared with the Lab-DR4 strain.

The expression levels of protease $m 1$ zinc metalloprotease in five strains with different levels of DM resistance were shown in Fig. 4. With the increase of resistance levels in lab strains $(\mathrm{DS}=0.04, \mathrm{DR} 1=0.31$, $\mathrm{DR} 2=0.85, \quad \mathrm{DR} 3=3.03, \quad \mathrm{DR} 4=3.43), \quad$ transcriptional expressions of the gene gradually decreased (Fig. 4a).
When the $\mathrm{LC}_{50}$ was increased 75-fold, the transcriptional expression was decreased by approximately $97 \%\left(t_{(4)}=50.53, P<0.0001\right)$. We used the correlation analysis and found a significant negative correlation between relative quantification of protease $m 1$ zinc metalloprotease $(Y)$ and the $\mathrm{LC}_{50}(X)\left(r_{(\mathrm{xy})}=-0.974, P=0.005\right.$, Fig. $4 \mathrm{~b})$. The correlation coefficient indicated that the gene may play a role in the development of deltamethrin resistance in $C x$. pipiens pallens.

We then expanded mosquito samples to identify the expression level in field-collected strains. The protease $m 1$ zinc metalloprotease transcriptional expression was analyzed in SH, GD, HM, JN, PY field-collected populations, separately (Fig. 5). In PY population, the comparison between susceptible and resistant mosquitoes indicated no significant differences in transcription. But for the other populations, the gene was down-regulated in the resistant strain of the populations compared to the corresponding susceptible populations $(0.50$-fold in $\mathrm{SH}, 0.41$-fold in GD, 0.88-fold in HM, 0.64-fold in JN, respectively), which was consistent with the result of the

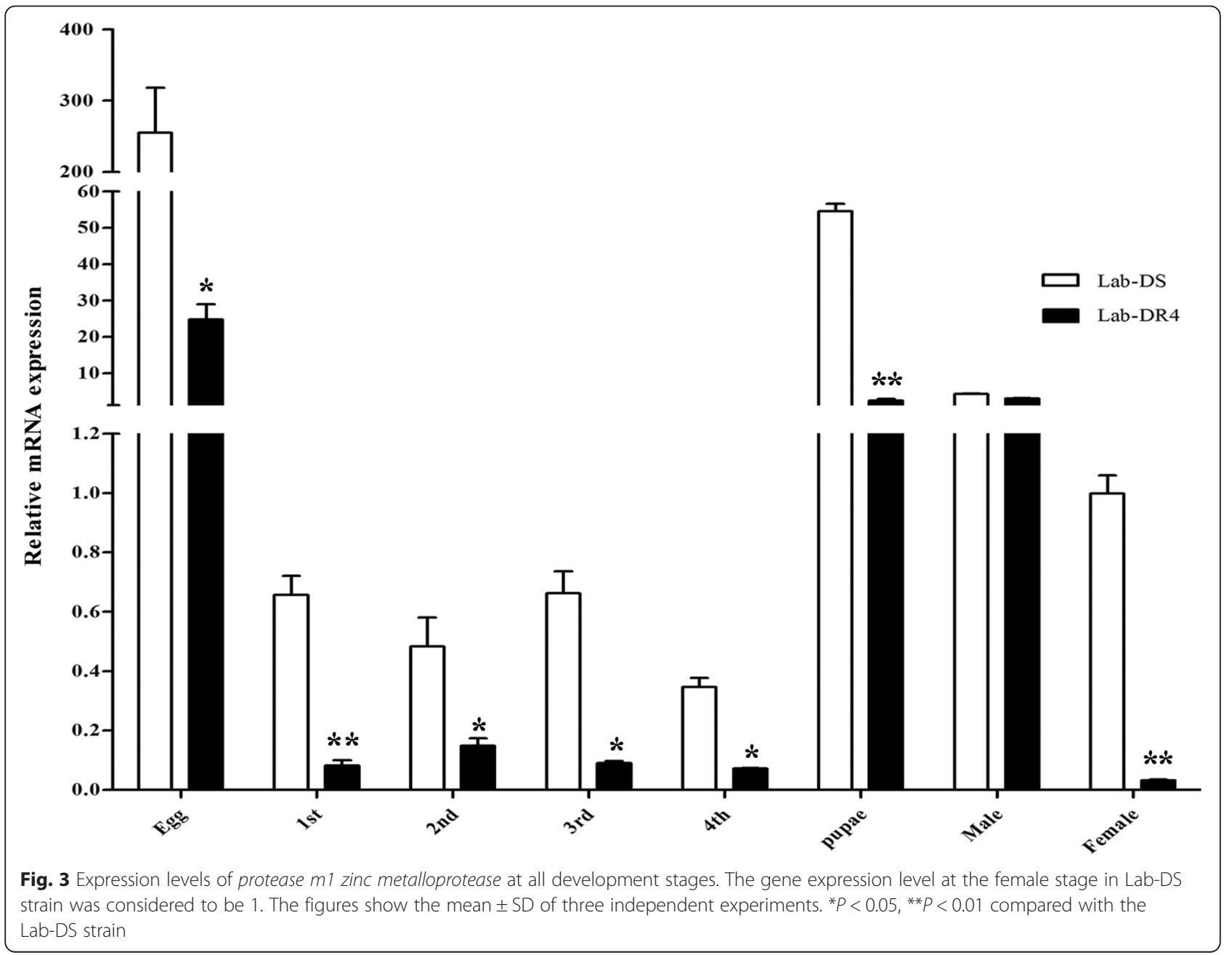




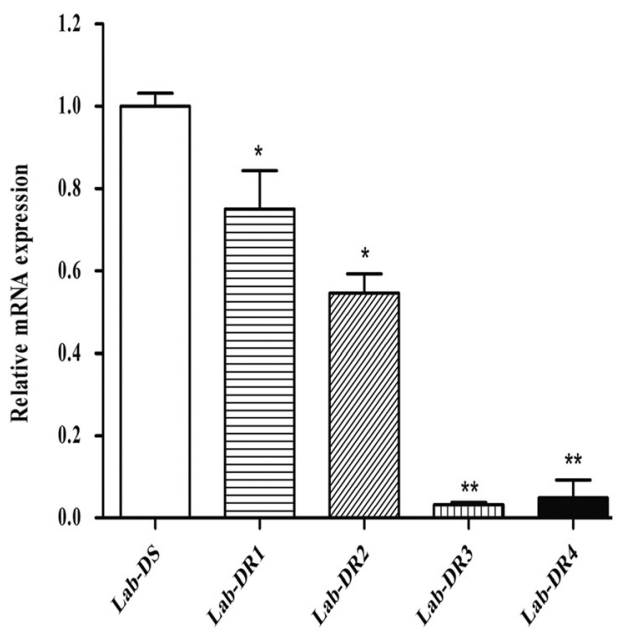

A

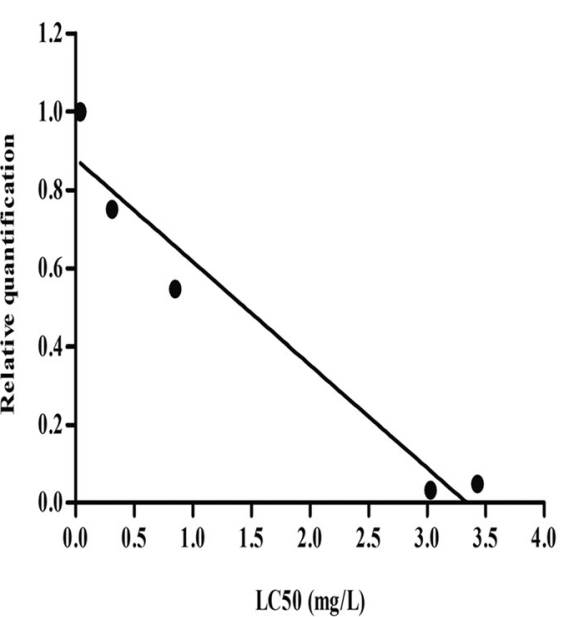

B

Fig. 4 a Expression profiles of protease $m 1$ zinc metalloprotease transcripts in five strains with different deltamethrin resistance levels. The results were shown as the mean \pm S.E. Significant differences indicated by ${ }^{*}(P<0.05)$ and ${ }^{* *}(P<0.01)$ were compared with the Lab-DS strain. $\mathbf{b}$ The relationship between protease $m 1$ zinc metalloprotease transcriptional levels and deltamethrin resistance of Culex pipiens pallens. The $Y$-axis was the ratio of the gene expression in Lab-DR mosquitoes compared with that in Lab-DS mosquitoes, $r=-0.97, P<0.05$

lab strains. The gene may be used as a potential genetic marker to monitor the pyrethroid resistance.

We compared the expression of protease $m 1$ zinc metalloprotease at different life stages (egg, larvae, pupae, adult males and females) between the Lab-DS and Lab-DR4 strains, in five lab strains with different DM-resistance levels, and in five different field-collected strains. The results showed that the gene maintained a high expression in the susceptible strain. Notably, the regression analysis performed to determine the relationship of transcriptional levels of protease $m 1$ zinc metalloprotease and the $\mathrm{LC}_{50}$ of deltamethrin

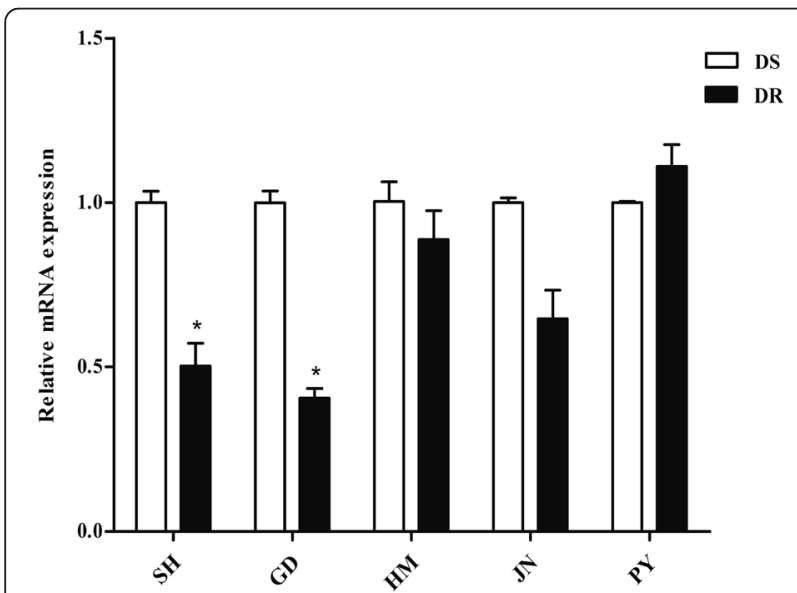

Fig. 5 The expression analysis of protease $m 1$ zinc metalloprotease in field-collected strains of Culex pipiens pallens. The results were shown as the mean \pm S.E. Significant differences indicated by $*(P<0.05)$ were compared with the corresponding susceptible strain revealed a statistically significant negative relationship $\left(r_{(\mathrm{xy})}=-0.974, P=0.005\right)$. And it was reported that the midgut transcript levels of two APN genes (AAEL008158 and AAEL008162, belonging to the peptidase family M1), were significantly reduced in insecticide resistant Aedes aegypti larvae compared to the susceptible ones [22]. Aminopeptidases are widely distributed throughout the insect kingdom. In our lab strains of $C x$. pipiens pallens, the gene expression of protease $m 1$ zinc metalloprotease was in various structures but mainly in legs and head (Fig. 6b), which suggested the mechanism of this zinc metalloprotease involved in the DM resistance was different from the mechanism of APNs involved in Bti resistance $[47,48]$.

The relationship of mRNA level analysis between CYP6CP1 and protease $m 1$ zinc metalloprotease

In our study, the expression level of CYP6CP1 was upregulated while the level of protease $m 1$ zinc metalloprotease was down-regulated in the DM-resistance strain of $C x$. pipiens pallens compared with the susceptible one. And the expression level of $C Y P 6 C P 1$ was up-regulated when protease $m 1$ zinc metalloprotease was knocked down in susceptible adults $\left(t_{(4)}=4.054, P=0.015\right.$ ) (Fig. 7). We detected that knockdown of CYP6CP1 increased the sensitivity of mosquitoes to DM (Additional file 6). Meanwhile, we analyzed the expression profile of CYP6CP1 in various structures and detected the gene was mainly in legs and head, which was similar to the expression of protease $m 1$ zinc metalloprotease (Fig. 6).

It has been proved that increased P450-mediated detoxification is a major mechanism of pyrethroid 

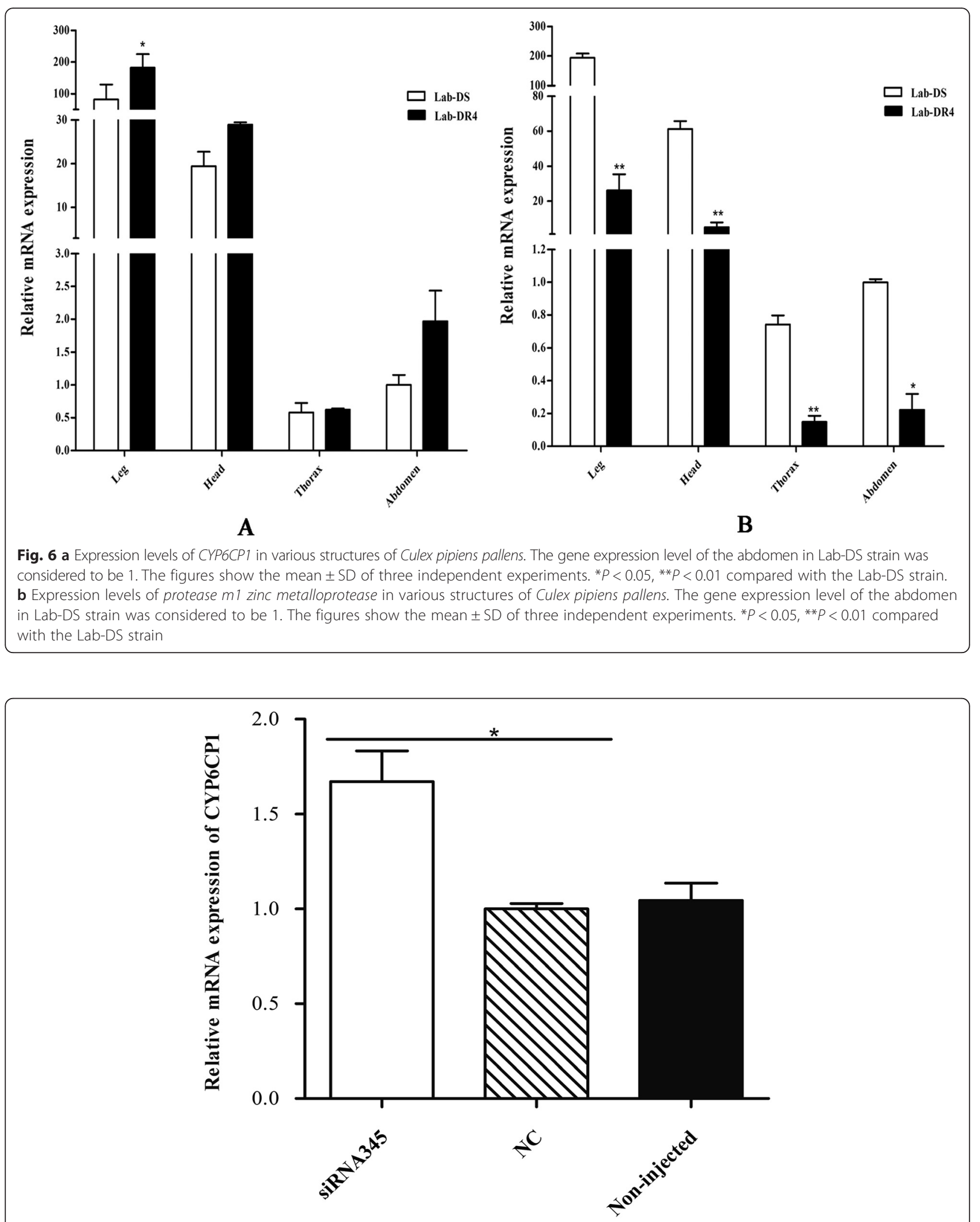

Fig. 7 Relative expression of CYP6CP1 in mosquitoes injected with dsRNA of protease $m 1$ zinc metalloprotease. The relative gene expression of CYP6CP1 along the $Y$-axis was the ratio of the gene expression in siRNA345-injected (dsRNA of protease $m 1$ zinc metalloprotease) mosquitoes compared with that in NC-injected mosquitoes. The results were shown as the mean \pm S.E. Significant differences were indicated by $*(P<0.05)$ 
resistance in insects $[49,50]$, but the regulatory mechanisms of P450 up-regulation are still unclear. Metalloproteases play essential roles in regulation of metastasis, signal-transduction, spatial proteolytic activity and modulation of protein-protein and cell-cell interactions [51-53]. Mosha et al. showed that metalloprotease could be a regulatory factor for CYP2J2 in the hyperhomocysteinemia [54]. It was also reported that overexpression of CYP4A11 significantly induced invasion and expression of the MMP-9 (MMPs are classified as zinc-dependent proteinases) [55]. In our study, we detected that knockdown of protease $m 1$ zinc metalloprotease increased the expression of CYP $6 C P 1$. So we speculated that protease $m 1$ zinc metalloprotease may be involved in DM resistance by affecting the transcriptional level of CYP6CP1. Further experiments are needed to investigate the regulatory role of protease $m 1$ zinc metalloprotease.

\section{Conclusions}

In summary, protease $m 1$ zinc metalloprotease was identified as a novel DM-resistance-associated gene using transcription analysis and RNAi, from the flanking sequences of the marker L3A8.177 in the genome sequence of the closely related $C x$. pipiens quinquefasciatus. This study has shed new light on the potential function of protease $m 1$ zinc metalloprotease in DM resistance and its regulatory function on resistancerelated $\mathrm{P} 450$ gene expression. However, the entire regulatory pathway remains largely unclear. The work not only lays a foundation for identification of DM resistance genes in $C x$. pipiens pallens by a genetic marker, but also identification of a novel deltamethrin-resistance-associated gene.

\section{Additional files}

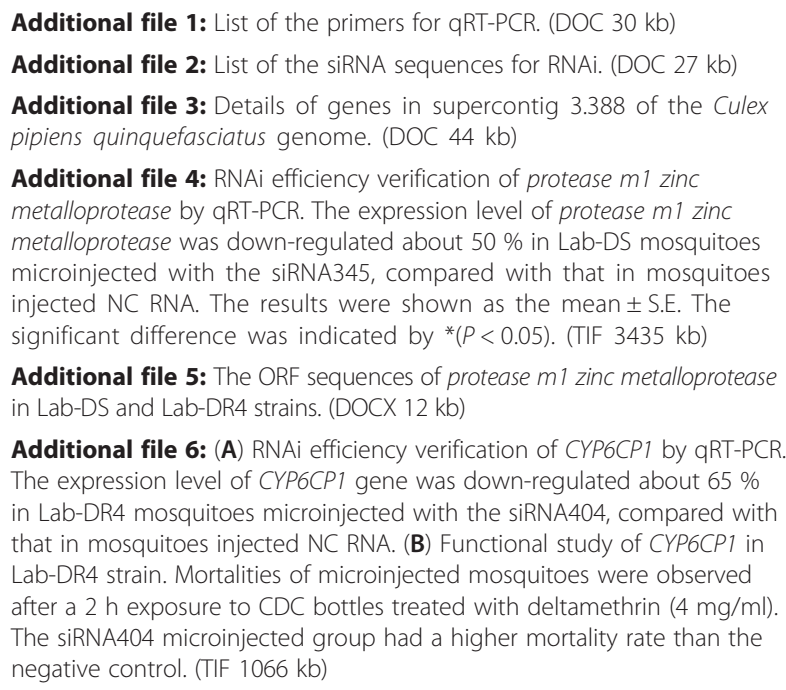

Additional file 5: The ORF sequences of protease $m 1$ zinc metalloprotease in Lab-DS and Lab-DR4 strains. (DOCX 12 kb)

Additional file 6: (A) RNAi efficiency verification of CYP6CP1 by qRT-PCR. The expression level of CYP6CP1 gene was down-regulated about $65 \%$ in Lab-DR4 mosquitoes microinjected with the siRNA404, compared with that in mosquitoes injected NC RNA. (B) Functional study of CYP6CP1 in Lab-DR4 strain. Mortalities of microinjected mosquitoes were observed after a $2 \mathrm{~h}$ exposure to CDC bottles treated with deltamethrin $(4 \mathrm{mg} / \mathrm{ml})$. The siRNA404 microinjected group had a higher mortality rate than the negative control. (TIF $1066 \mathrm{~kb}$ )

\section{Competing interests}

The authors declare that they have no competing interests.

\section{Authors' contributions}

FFZ, BS and CLZ conceived and designed the study. FFZ, QG, YS, DZ, MXH, $\mathrm{HXH}, \mathrm{BQL}, \mathrm{MMT}, \mathrm{XML}, \mathrm{XXL}$ and $L M$ performed the experiments. FFZ, BS and $\mathrm{CLZ}$ analyzed the data and wrote the paper. All authors read and approved the final version for submission.

\section{Acknowledgements \\ This work was supported by the National Institutes of Health of US (NIH) (Grant No. 2R01Al075746), the National Natural Science Foundation of China (Grant No. 81171900 and 81101279), the National S \& T Major Program (Grant No. 2012ZX10004-219 and 2012ZX10004-220), the Specialized Research Fund for the Doctoral Program of Higher Education of China (Grant No.20113234120007), the Natural Science Foundation of Jiangsu Province (Grant No.81101279), and the Priority Academic Program Development of Jiangsu Higher Education Institutions.}

Received: 25 January 2016 Accepted: 12 March 2016

Published online: 23 March 2016

\section{References}

1. WHO. Vector-borne Diseases. Media Centre: WHO; 2014.

2. Reiner Jr RC, Perkins TA, Barker CM, Niu T, Chaves LF, Ellis AM, George DB, Le Menach A, Pulliam JR, Bisanzio D et al. A systematic review of mathematical models of mosquito-borne pathogen transmission: 1970-2010. J R Soc Interface. 2013;10(81):20120921.

3. Barbosa S, Black WC, Hastings I. Challenges in estimating insecticide selection pressures from mosquito field data. PLoS Negl Trop Dis. 2011;5(11), e1387.

4. WHO. Malaria. Media Centre: WHO; 2016.

5. WHO. Global plan for insecticide resistance management in malaria vectors. Geneva: WHO; 2012

6. Kumar S, Thomas A, Sahgal A, Verma A, Samuel T, Pillai MK. Effect of the synergist, piperonyl butoxide, on the development of deltamethrin resistance in yellow fever mosquito, Aedes aegypti L. (Diptera: Culicidae). Arch Insect Biochem Physiol. 2002;50(1):1-8.

7. Saavedra-Rodriguez K, Urdaneta-Marquez L, Rajatileka S, Moulton M, Flores AE, Fernandez-Salas I, Bisset J, Rodriguez M, McCall PJ, Donnelly MJ et al. A mutation in the voltage-gated sodium channel gene associated with pyrethroid resistance in Latin American Aedes aegypti. Insect Mol Biol. 2007;16(6):785-98.

8. Bonizzoni M, Ochomo E, Dunn WA, Britton M, Afrane Y, Zhou G, Hartsel J, Lee MC, Xu J, Githeko A et al. RNA-seq analyses of changes in the Anopheles gambiae transcriptome associated with resistance to pyrethroids in Kenya: identification of candidate-resistance genes and candidate-resistance SNPS. Parasit Vectors. 2015:8:474.

9. Yanola J, Chamnanya S, Lumjuan N, Somboon P. Insecticides resistance in the Culex quinquefasciatus populations from northern Thailand and possible resistance mechanisms. Acta Trop. 2015;149:232-8.

10. Lv Y, Wang W, Hong S, Lei Z, Fang F, Guo Q, Hu S, Tian M, Liu B, Zhang D et al. Comparative transcriptome analyses of deltamethrin-susceptible and -resistant Culex pipiens pallens by RNA-seq. Mol Gen Genomics. 2015. doi:10.1007/s00438-015-1109-4.

11. Li T, Liu L, Zhang L, Liu N. Role of G-protein-coupled receptor-related genes in insecticide resistance of the mosquito, Culex quinquefasciatus. Sci Rep. 2014;4:6474

12. Maestre-Serrano R, Gomez-Camargo D, Ponce-Garcia G, Flores AE. Susceptibility to insecticides and resistance mechanisms in Aedes aegypti from the Colombian Caribbean Region. Pestic Biochem Physiol. 2014;116:63-73.

13. Zou F, Chen C, Zhong D, Shen B, Zhang D, Guo Q, Wang W, Yu J, Lv Y, Lei $Z$ et al. Identification of QTLs conferring resistance to Deltamethrin in Culex pipiens pallens. PLoS One. 2015;10(10), e0140923.

14. Hickner PV, Debruyn B, Lovin DD, Mori A, Saski CA, Severson DW. Enhancing genome investigations in the mosquito Culex quinquefasciatus via BAC library construction and characterization. BMC Res Notes. 2011;4:358.

15. Kothera L, Zimmerman EM, Richards CM, Savage HM. Microsatellite characterization of subspecies and their hybrids in Culex pipiens complex (Diptera: Culicidae) mosquitoes along a north-south transect in the central United States. J Med Entomol. 2009;46(2):236-48. 
16. Arensburger P, Megy K, Waterhouse RM, Abrudan J, Amedeo P, Antelo B, Bartholomay L, Bidwell S, Caler E, Camara F et al. Sequencing of Culex quinquefasciatus establishes a platform for mosquito comparative genomics. Science. 2010;330(6000):86-8.

17. Lyons RE, Dierens LM, Tan SH, Preston NP, Li Y. Characterization of AFLP markers associated with growth in the Kuruma prawn, Marsupenaeus japonicus, and identification of a candidate gene. Mar Biotechnol. 2007;9(6):712-21.

18. Hemingway J, Hawkes NJ, McCarroll L, Ranson $\mathrm{H}$. The molecular basis of insecticide resistance in mosquitoes. Insect Biochem Mol Biol. 2004;34(7):653-65.

19. Brownlees J, Williams $\mathrm{CH}$. Peptidases, peptides, and the mammalian blood-brain barrier. J Neurochem. 1993;60(3):793-803.

20. Pardo A, Selman M. Matrix metalloproteases in aberrant fibrotic tissue remodeling. Proc Am Thorac Soc. 2006;3(4):383-8.

21. Paris $M$, Despres $L$. Identifying insecticide resistance genes in mosquito by combining AFLP genome scans and 454 pyrosequencing. Mol Ecol. 2012;21(7):1672-86.

22. Lee SB, Aimanova KG, Gill SS. Alkaline phosphatases and aminopeptidases are altered in a Cry 11Aa resistant strain of Aedes aegypti. Insect Biochem Mol Biol. 2014;54:112-21.

23. Wang W, Lv Y, Fang F, Hong S, Guo Q, Hu S, Zou F, Shi L, Lei Z, Ma K et al. Identification of proteins associated with pyrethroid resistance by iTRAQ-based quantitative proteomic analysis in Culex pipiens pallens. Parasit Vectors. 2015;8:95.

24. WHO. Test procedures for insecticide resistance monitoring in malaria. Geneva: WHO; 2013.

25. Zhou D, Hao S, Sun Y, Chen L, Xiong C, Ma L, Zhang D, Hong S, Shi L, Gong $M$ et al. Cloning and characterization of prophenoloxidase A3 (proPOA3) from Culex pipiens pallens. Comp Biochem Physiol B Biochem Mol Biol. 2012;162(4):57-65.

26. Fang F, Wang W, Zhang D, Lv Y, Zhou D, Ma L, Shen B, Sun Y, Zhu C. The cuticle proteins: a putative role for deltamethrin resistance in Culex pipiens pallens. Parasitol Res. 2015;114(12):4421-9.

27. Canales M, Naranjo V, Almazan C, Molina R, Tsuruta SA, Szabo MP, Manzano-Roman R, Perez de la Lastra JM, Kocan KM, Jimenez Ml et al. Conservation and immunogenicity of the mosquito ortholog of the tick-protective antigen, subolesin. Parasitol Res. 2009;105(1):97-111.

28. Leal WS, Choo YM, Xu P, da Silva CS, Ueira-Vieira C. Differential expression of olfactory genes in the southern house mosquito and insights into unique odorant receptor gene isoforms. Proc Natl Acad Sci U S A. 2013;110(46):18704-9.

29. Blandin S, Moita LF, Kocher T, Wilm M, Kafatos FC, Levashina EA. Reverse genetics in the mosquito Anopheles gambiae: targeted disruption of the Defensin gene. EMBO Rep. 2002;3(9):852-6.

30. Dong Y, Aguilar R, Xi Z, Warr E, Mongin E, Dimopoulos G. Anopheles gambiae immune responses to human and rodent Plasmodium parasite species. PLoS Pathog. 2006;2(6), e52.

31. Li T, Liu LN, Zhang L, Liu NN. Role of G-protein-coupled receptor-related genes in insecticide resistance of the mosquito, Culex quinquefasciatus. Sci Rep. 2014;4.

32. LV Y, Lei ZT, Hong SC, Wang WJ, Zhang DH, Zhou D, Sun Y, Ma L, Shen B, Zhu CL. Venom allergen 5 is associated with deltamethrin resistance in Culex pipiens pallens (Diptera: Culicidae). J Med Entomol. 2015;52(4):672-82.

33. Podio M, Rodriguez MP, Felitti S, Stein J, Martinez EJ, Siena LA, Quarin CL, Pessino SC, Ortiz JP. Sequence characterization, in silico mapping and cytosine methylation analysis of markers linked to apospory in Paspalum notatum. Genet Mol Biol. 2012;35(4):827-37.

34. David JP, Strode C, Vontas J, Nikou D, Vaughan A, Pignatelli PM, Louis C, Hemingway J, Ranson H. The Anopheles gambiae detoxification chip: a highly specific microarray to study metabolic-based insecticide resistance in malaria vectors. Proc Natl Acad Sci U S A. 2005;102(11):4080-4.

35. Strode C, Wondji CS, David JP, Hawkes NJ, Lumjuan N, Nelson DR, Drane DR, Karunaratne SH, Hemingway J, Black WC et al. Genomic analysis of detoxification genes in the mosquito Aedes aegypti. Insect Biochem Mol Biol. 2008;38(1):113-23.

36. David JP, Faucon F, Chandor-Proust A, Poupardin R, Riaz MA, Bonin A, Navratil V, Reynaud S. Comparative analysis of response to selection with three insecticides in the dengue mosquito Aedes aegypti using mRNA sequencing. BMC Genom. 2014;15:174.

37. Yang T, Liu N. Genome analysis of cytochrome P450s and their expression profiles in insecticide resistant mosquitoes, Culex quinquefasciatus. PLoS One. 2011;6(12), e29418.
38. Wondji CS, Irving H, Morgan J, Lobo NF, Collins FH, Hunt RH, Coetzee M, Hemingway J, Ranson H. Two duplicated P450 genes are associated with pyrethroid resistance in Anopheles funestus, a major malaria vector. Genome Res. 2009;19(3):452-9.

39. Djouaka RF, Bakare AA, Coulibaly ON, Akogbeto MC, Ranson H, Hemingway J, Strode C. Expression of the cytochrome P450s, CYP6P3 and CYP6M2 are significantly elevated in multiple pyrethroid resistant populations of Anopheles gambiae s.s. from Southern Benin and Nigeria. BMC Genomics. 2008;9:538.

40. Liu N, Li T, Reid WR, Yang T, Zhang L. Multiple Cytochrome P450 genes: their constitutive overexpression and permethrin induction in insecticide resistant mosquitoes, Culex quinquefasciatus. PLoS One. 2011;6(8), e23403.

41. Hooper NM. Families of zinc metalloproteases. FEBS Lett. 1994;354(1):1-6.

42. Gomez MA, Contreras I, Halle M, Tremblay ML, McMaster RW, Olivier M. Leishmania GP63 alters host signaling through cleavage-activated protein tyrosine phosphatases. Sci Signal. 2009;2(90):ra58.

43. Shio MT, Christian JG, Jung JY, Chang KP, Olivier M. PKC/ROS-Mediated NLRP3 inflammasome activation is attenuated by Leishmania zinc-metalloprotease during infection. PLoS Negl Trop Dis. 2015;9(6), e0003868.

44. Rajagopal R, Sivakumar S, Agrawal N, Malhotra P, Bhatnagar RK. Silencing of midgut aminopeptidase $\mathrm{N}$ of Spodoptera litura by double-stranded RNA establishes its role as Bacillus thuringiensis toxin receptor. J Biol Chem. 2002:277(49):46849-51.

45. Sivakumar S, Rajagopal R, Venkatesh GR, Srivastava A, Bhatnagar RK. Knockdown of aminopeptidase-N from Helicoverpa armigera larvae and in transfected Sf21 cells by RNA interference reveals its functional interaction with Bacillus thuringiensis insecticidal protein Cry1Ac. J Biol Chem. 2007;282(10):7312-9.

46. Zhang R, Hua G, Urbauer JL, Adang MJ. Synergistic and inhibitory effects of aminopeptidase peptides on Bacillus thuringiensis Cry11Ba toxicity in the mosquito Anopheles gambiae. Biochemistry. 2010;49(39):8512-9.

47. Chen J, Likitvivatanavong S, Aimanova KG, Gill SS. A 104 kDa Aedes aegypti aminopeptidase $\mathrm{N}$ is a putative receptor for the Cry11Aa toxin from Bacillus thuringiensis subsp. israelensis. Insect Biochem Mol Biol. 2013;43(12):1201-8.

48. Luo YX, Cui J, Wang L, Chen DK, Peng JS, Lan P, Huang MJ, Huang YH, Cai $\mathrm{SR}$, Hu KH et al. Identification of cancer-associated proteins by proteomics and downregulation of beta-tropomyosin expression in colorectal adenoma and cancer. Proteomics Clin Appl. 2009;3(12):1397-406.

49. Scott JG, Yoshimizu MH, Kasai S. Pyrethroid resistance in Culex pipiens mosquitoes. Pestic Biochem Physiol. 2015;120:68-76.

50. David JP, Ismail HM, Chandor-Proust A, Paine MJ. Role of cytochrome P450s in insecticide resistance: impact on the control of mosquito-borne diseases and use of insecticides on Earth. Philos Trans R Soc, B Bio Sci. 2013;368(1612):20120429. doi:10.1098/rstb.2012.0429.

51. Chang C, Werb Z. The many faces of metalloproteases: cell growth, invasion, angiogenesis and metastasis. Trends Cell Biol. 2001;11(11):S37-43.

52. Cerda-Costa N, Xavier Gomis-Ruth F. Architecture and function of metallopeptidase catalytic domains. Protein Sci. 2014;23(2):123-44.

53. Ellis V, Murphy G. Cellular strategies for proteolytic targeting during migration and invasion. Febs Lett. 2001;506(1):1-5.

54. Mosha KS, Zeldin DC, Sithu SD, Sen U, Tyagi N, Kumar M, Hughes WM, Metreveli N, Rosenberger DSE, Singh M et al. Cytochrome p450 (CYP) 2 J2 gene transfection attenuates MMP-9 via inhibition of NF-kappa beta in hyperhomocysteinemia. J Cell Physiol. 2008;215(3):771-81.

55. Yu W, Chen L, Yang YQ, Falck JR, Guo AM, Li Y, Yang J. Cytochrome P450 omega-hydroxylase promotes angiogenesis and metastasis by upregulation of VEGF and MMP-9 in non-small cell lung cancer. Cancer Chemother Pharmacol. 2011;68(3):619-29. 